\title{
Common and Dissociable Neural Activity After Mindfulness-Based Stress Reduction and Relaxation Response Programs
}

\section{Citation}

Sevinc, Gunes, Britta K. Hölzel, Javeria Hashmi, Jonathan Greenberg, Adrienne McCallister, Michael Treadway, Marissa L. Schneider, Jeffery A. Dusek, James Carmody, and Sara W. Lazar. 2018. "Common and Dissociable Neural Activity After Mindfulness-Based Stress Reduction and Relaxation Response Programs." Psychosomatic Medicine 80 (5): 439-451. doi:10.1097/ PSY.0000000000000590. http://dx.doi.org/10.1097/PSY.0000000000000590.

\section{Published Version}

doi:10.1097/PSY.0000000000000590

\section{Permanent link}

http://nrs.harvard.edu/urn-3:HUL.InstRepos:37298401

\section{Terms of Use}

This article was downloaded from Harvard University's DASH repository, and is made available under the terms and conditions applicable to Other Posted Material, as set forth at http:// nrs.harvard.edu/urn-3:HUL.InstRepos:dash.current.terms-of-use\#LAA

\section{Share Your Story}

The Harvard community has made this article openly available.

Please share how this access benefits you. Submit a story.

Accessibility 


\title{
Common and Dissociable Neural Activity After Mindfulness-Based Stress Reduction and Relaxation Response Programs
}

\author{
Gunes Sevinc, PhD, Britta K. Hölzel, PhD, Javeria Hashmi, PhD, Jonathan Greenberg, PhD, \\ Adrienne McCallister, DO, Michael Treadway, PhD, Marissa L. Schneider, BA, Jeffery A. Dusek, PhD, \\ James Carmody, PhD, and Sara W. Lazar, PhD
}

\begin{abstract}
Objective: We investigated common and dissociable neural and psychological correlates of two widely used meditation-based stress reduction programs.

Methods: Participants were randomized to the Relaxation Response (RR; $n=18 ; 56 \%$ female) or the Mindfulness-Based Stress Reduction (MBSR; $n=16 ; 56 \%$ female) programs. Both programs use a "bodyscan" meditation; however, the RR program explicitly emphasizes physical relaxation during this practice, whereas the MBSR program emphasizes mindful awareness with no explicit relaxation instructions. After the programs, neural activity during the respective meditation was investigated using functional magnetic resonance imaging.

Results: Both programs were associated with reduced stress (for RR, from $14.1 \pm 6.6$ to $11.3 \pm 5.5$ [Cohen's $d=0.50$; for MBSR, from $17.7 \pm 5.7$ to $11.9 \pm 5.0$ [Cohen's $d=1.02]$ ). Conjunction analyses revealed functional coupling between ventromedial prefrontal regions and supplementary motor areas $(p<.001)$. The disjunction analysis indicated that the RR bodyscan was associated with stronger functional connectivity of the right inferior frontal gyrus — an important hub of intentional inhibition and control—with supplementary motor areas $(p<.001$, family-wise error [FWE] rate corrected). The MBSR program was uniquely associated with improvements in self-compassion and rumination, and the within-group analysis of MBSR bodyscan revealed significant functional connectivity of the right anterior insula - an important hub of sensory awareness and salience - with pregenual anterior cingulate during bodyscan meditation compared with rest ( $p=.03$, FWE corrected).

Conclusions: The bodyscan exercises in each program were associated with both overlapping and differential functional coupling patterns, which were consistent with each program's theoretical foundation. These results may have implications for the differential effects of these programs for the treatment of diverse conditions.
\end{abstract}

Key words: meditation, mindfulness, mindfulness-based stress reduction program, relaxation response, bodyscan.

\section{INTRODUCTION}

$\mathbf{T}$ he use of mind-body techniques as complementary medical interventions has been increasing dramatically (1). Most meditation-based clinical programs are modeled on one of two well-known stress reduction courses - the relaxation response (RR) program developed by Herbert Benson (2-4) or the mindfulness-based stress reduction (MBSR) program developed
ACC $=$ anterior cingulate cortex, alNS $=$ anterior insula, $\mathbf{C O N N}=$ Connectivity Toolbox, FFMQ = Five Facet Mindfulness Questionnaire, $\mathbf{F W E}=$ family-wise error rate, $\mathbf{M B S R}=$ mindfulness-based stress reduction, $\mathbf{p A C C}=$ pregenual anterior cingulate cortex, $\mathbf{P S S}=$ Perceived Stress Scale, $\mathbf{r I F G}=$ right inferior frontal gyrus, $\mathbf{R} \mathbf{R}=$ relaxation response, $\mathbf{S M A}=$ supplementary motor area, $\mathbf{S P M}=$ Statistical Parametric Mapping, $\mathbf{T R}=$ repetition time, $\mathbf{v M P F C}=$ ventral medial frontal cortex

\footnotetext{
SDC Supplemental Content

From the Department of Psychiatry (Sevinc, Hölzel, Greenberg, McCallister, Schneider, Lazar), Massachusetts General Hospital, Harvard Medical School, Boston, Massachusetts; Department of Neuroradiology, Klinikum rechts der Isar (Hölzel), Technical University of Munich, Munich, Germany; Department of Anesthesia, Pain Management and Perioperative Medicine (Hashmi), Dalhousie University, Dalhousie University, Halifax, Canada; Department of Osteopathic Manipulative Medicine (McCallister), Berkshire Medical Center, Pittsfield, Massachusetts; Department of Psychology (Treadway), Emory University, Atlanta, Georgia; Kripalu Center for Yoga and Health (Dusek), Stockbridge; Benson-Henry Institute for Mind Body Medicine (Dusek), Massachusetts General Hospital, Boston; and University of Massachusetts Medical School (Carmody), Worcester, Massachusetts.

Address correspondence to Gunes Sevinc, PhD, Department of Psychiatry, Division of Psychiatric Neuroscience, Massachusetts General Hospital, Harvard Medical School, 120 2nd Ave, Charlestown, MA 02129. E-mail: gkayaci@gmail.com; gsevinc@mgh.harvard.edu

Received for publication July 31, 2017; revision received March 5, 2018.

DOI: 10.1097/PSY.0000000000000590

Copyright $\odot 2018$ The Author(s). Published by Wolters Kluwer Health, Inc. on behalf of the American Psychosomatic Society. This is an open-access article distributed under the terms of the Creative Commons Attribution-Non Commercial-No Derivatives License 4.0 (CCBY-NC-ND), where it is permissible to download and share the work provided it is properly cited. The work cannot be changed in any way or used commercially without permission from the journal.
} 
by Jon Kabat-Zinn (5-7). Both programs have been operating for more than 40 years, and their effectiveness has been validated with samples of patients with a wide variety of illnesses (8-13). Although both interventions are based on meditation, the scientific philosophies and meditative traditions upon which each is founded are distinct: the RR program emphasizes the induction of a relaxed physiological state hypothesized to be the opposite of the stress response, whereas the MBSR program is hypothesized to work by cultivating a particular nonjudgmental attitude termed "mindfulness." Consequently, MBSR does not necessarily encourage relaxation but posits that a form of "meta-relaxation" may arise from the nonjudgmental acceptance of any given body state. Given these similarities and implied theoretical differences, the goal of this study is to compare psychological changes and investigate putative common and dissociable neural activation patterns associated with each program. A finding of both common and divergent neural activation patterns could potentially have implications for how these programs are prescribed.

The different theoretical orientations are reflected in how each meditation technique is practiced. The difference is most apparent in the "bodyscan" meditation practice, during which attention is moved sequentially through the body. The general instructions in both programs are the same, but the RR program explicitly instructs participants to intentionally reduce arousal and muscle tension in each body area with the goal of decreasing sympathetic activation (14). Conversely, the MBSR program emphasizes paying attention to present moment sensory experience in each body area without trying to change anything and explicitly teaches participants that physical relaxation is not an aim of meditation practice (6). This differential emphasis on relaxation versus mindfulness applies to all the techniques taught in both programs. Indeed, the founders of each program argue that the specific meditation techniques used are less important than how these techniques are practiced. As such, although the RR program includes some instruction in mindfulness, it is a relatively minor component of the program and typically consists of instructions to be mindful of becoming more relaxed.

Despite their long history, these programs have never been directly compared in a single study, and so it has been impossible to compare and contrast their neural correlates and behavioral outcomes. Numerous studies of many different types of meditation have been performed and have found both overlapping and divergent results in terms of peripheral and autonomic physiology $(4,15-17)$, neural activation $(18,19)$, and psychological/cognitive (20-23) effects. However, some studies have used highly experienced practitioners, whereas others have used novices or clinical populations. Furthermore, a comparison of studies is confounded by issues related to selection bias (24), as well as differences in data collection and analysis techniques. Indeed, even studies that have specifically studied the neural effects of mindfulness meditation, which has been the most thoroughly investigated form to date, have found both overlapping and divergent results (25), which underscores the need for a direct comparison of meditation techniques using the same neuroimaging acquisition and analysis protocols to identify similarities and differences as to how these meditation techniques are actually performed.

The purpose of this study was specifically to address common and dissociable functional coupling patterns during meditation practice, rather than the longitudinal change in brain activity due to training. Therefore, the scanning was only performed at the posttraining time point because meditating in the scanner is challenging, and individuals often need extensive instruction and practice before they are able to achieve a meditative state. Thus, by scanning only after the programs, meditation-naïve participants had received sufficient training and experience to meditate in the scanner.

The current study investigates shared and divergent neural activation patterns modulated by each bodyscan meditation technique because it is the most similar practice between the two programs. As mentioned earlier, both RR and MBSR bodyscan meditations involve an awareness of bodily sensations. One of the main brain regions associated with such focused attention meditation and a present-centered awareness of the self $(26,27)$ is the ventral medial prefrontal cortex (vMPFC). Because presentcentered awareness of the self is shared across both types of meditation, we hypothesized that functional coupling pattern of the vMPFC would be common feature of both programs.

In terms of brain regions that might be unique to each meditation style, because we are specifically interested in dissociable neural activation patterns that would reflect differences in the theoretical orientation of each program, we selected seeds that might potentially reflect the differential intentions of "to relax" or "to be mindful." Although there have been no neuroimaging studies of the RR program, a study by Tang et al. (28) compared relaxation training, which was somewhat similar to the RR program, with integrative mind-body training. The researchers demonstrated that relaxation training produced differential activation in the lateral prefrontal cortex, a region implicated in cognitive control, response inhibition, and the selection of information (29-31). They concluded that this region might be involved in effortful control, which is critical to create and maintain a relaxed state. Therefore, we hypothesized that the functional coupling pattern of this region might be different during the two styles of body scan.

Conversely, although there have been many neuroimaging studies of mindfulness meditation, most of these have used longterm practitioners of various traditions $(18,25)$. There have been no studies of the mindful bodyscan; however, two studies have identified the insula as a primary region activated by recent MBSR graduates $(26,32)$. The insula has been repeatedly associated with mindfulness meditation in both structural $(33,34)$ and functional studies (35-37). Although sensory feedback is integral to both types of bodyscan, only the MBSR program emphasizes deliberate awareness of these sensations, whereas the RR program focuses more on elicitation of relaxation regardless of the current bodily sensations. Relying on anterior insula's (aINS) role in somatosensory conscious awareness (38) and receiving moment-to-moment inputs from a variety of somatic and sensory centers (39), as well as on the MBSR program's differential theoretical orientation, which strongly emphasizes sensory awareness, we hypothesized that the functional coupling of aINS would also differentiate MBSR from RR.

In line with their theoretical premises, each program has predominantly used different psychological outcome measures to assess therapeutic success. A direct comparison of the two programs using a single set of measures may provide important insights into the differences and similarities between them. In the current study, we address this need by additionally exploring similarities and differences in psychological outcomes. Both programs aim to decrease stress, and mindfulness, self-compassion, and rumination are often cited as key mechanisms through which the MBSR 
program works $(22,23,40)$. Any group differences in these metrics, together with the investigation of common and divergent neural coupling patterns during meditation practice, could help identify behavioral outcomes and neural correlates, which may be uniquely associated with each program, and contribute to our understanding how specific meditation programs may differentially influence outcomes. Such an understanding has potential implications for clinical practice because the various psychological outcomes associated with each type of program might be differentially beneficial for certain conditions.

\section{METHODS}

\section{Participants}

Seventy-four participants were assessed for eligibility, and 60 who met the inclusion criteria were enrolled in cohorts of 15 (see Appendix A for CONSORT flow diagram, Supplemental Digital Content, http://links.lww.com/ PSYMED/A474). Sample size was determined based on our unpublished pilot study of long-term Vipassana meditation and RR practitioners. Participants were recruited in six clusters/cohorts and randomized sequentially based on time of enrollment. All recruiting occurred during the periods from January to March and from August to October, and classes took place either between March 2010 and May 2010 or October 2010 and December 2010. The order of classes was randomized (three cohorts per arm) by coin flip. Two MBSR classes occurred in the spring and one in the fall. One RR class took place in the spring and two were in the fall. There were no differences between the cohorts in terms of the perceived stress levels either at pre $(p=.16)$ or at post $(p=.27)$ time points. Ten individuals dropped out before baseline testing, and 50 individuals began the program (RR: $n=22$, MBSR: $n=28$ ); 40 completed at least one behavioral measure at the posttesting time point (RR: $n=20$, MBSR: $n=20$ ). Useable brain data were available from 34 participants (RR: $n=16,9$ female, mean age $=$ $39.87 \pm 10.29$ years; MBSR: $n=18,10$ female, mean age $=$ $37.47 \pm 9.14$ years). No adverse events were reported during the study. Six of the missing cases for brain analysis were due to corruption of the raw data during storage and/or data transfer, and thus were not related to any aspect of the programs. The other 10 dropped out of the study due to other time commitments. The groups did not differ demographically (see Table 1). Left-handed individuals, those taking medications that alter cerebral blood flow or metabolism, or those who had major illnesses that would interfere with participation in the study were excluded and individuals who met standard magnetic resonance imaging (MRI) exclusion criteria (e.g., having metallic implants) were excluded, as were those with a history of head trauma. Participants were required to have had no more than 4 hours of meditation or yoga practice of any tradition in their lifetime. The age inclusion range was 22 to 55 years to minimize the effect of advanced age on brain function (41). Study staff was not blind to allocation, yet participants were blind to study objectives and to the composition of the courses. They were told only that there were two stress reduction courses (Program "A" and "B"); no other information on either program was given. Both program providers were blind to study hypotheses. Questionnaires were collected via a secure website. Data remained de-identified with regard to group assignment until statistical analyses were conducted. The Massachusetts General Hospital's institutional review board approved all study procedures, and all participants signed a written consent form. Participants were compensated for their time and also were reimbursed for parking when needed. The study was registered at ClinicalTrials.gov with the identifier number of NCT00625807.

\section{Experimental Protocol}

\section{Stress Reduction Programs}

The two programs differ slightly in how they are typically taught in healthcare settings. Therefore, we modified both programs to match them in terms of contact hours with the teacher and amount of home meditation practice assigned (see hereinafter). Other than these changes, the content of each program was the same as typically taught in the clinic. Both groups received eight weekly, 2-hour group-training sessions and were instructed to practice for $20 \mathrm{~min} / \mathrm{d}$ at home with guided recordings. Both programs included group exercises and interactions about the challenges and achievements experienced in the process of integrating mindfulness/relaxation into their lives and into stressful situations encountered throughout the day.

\section{RR course}

Typically, this is a 10 -week program that meets weekly for 2 hours. Classes 8 and 9 were removed to fit the 8 -week format of the study. Over the 8 -week program, four different techniques for eliciting the RR were taught to participants: guided relaxing bodyscan, focus word (mental repetition of a word, sound, or phrase), breath counting, and breath awareness. One single 20-minute guided meditation session that included all four techniques was used throughout the entire duration of the program. Janet Fronk who has more than 20 years of experience teaching the RR program at the Benson/Henry Mind-Body Medical Institute taught the RR course.

\section{MBSR course}

Typically, this is an 8-week program that meets weekly for 2.5 hours and also includes an "all-day" retreat after the sixth class. To fit the constraints of the study, classes were shortened to 2 hours and the all-day retreat was not included. Typically, the course enrolls 25 to 30 participants and the

TABLE 1. Baseline Characteristics

\begin{tabular}{|c|c|c|c|c|c|c|}
\hline & RR & MBSR & Statistical Test Value & $p$ & $\mathrm{Se}$ & Cohen's $d$ \\
\hline Sample size & 22 & 28 & & & & \\
\hline Age, y & $42.1 \pm 11.5$ & $35.3 \pm 9.3$ & $t=-2.17$ & .04 & 2.44 & -0.65 \\
\hline (Neuroimaging subcohort) & $39.9 \pm 10.3$ & $37.5 \pm 9.1$ & $t=-0.71$ & .48 & 1.68 & -0.25 \\
\hline Sex, $M / F$ & $7 / 15$ & 10/17 & $\chi(1)=0.70$ & .62 & & \\
\hline Education, y & $16.7 \pm 1.4$ & $16.6 \pm 2.2$ & $t=-0.21$ & .83 & 0.31 & -0.05 \\
\hline Home practice (formal) & $805.6 \pm 410.5$ & $754.0 \pm 354.2$ & $t=-0.45$ & .65 & 89.58 & -0.19 \\
\hline Number of classes attended & $6.7 \pm 1.6$ & $5.9 \pm 1.8$ & $t=-1.66$ & .10 & 0.35 & -0.47 \\
\hline \multicolumn{7}{|l|}{ Holmes-Rahe } \\
\hline Pre & $3.09 \pm 2.4$ & $3.3 \pm 2.9$ & $t=-0.27$ & .79 & 0.51 & -0.08 \\
\hline Post & $3.1 \pm 3.4$ & $3.6 \pm 3.4$ & $t=0.59$ & .56 & 0.54 & 0.14 \\
\hline
\end{tabular}

$\mathrm{RR}=$ relaxation response MBSR $=$ mindfulness-based stress reduction 
2.5 hours provides time for class participation from everyone. Because the study enrolled just 15 people per cohort, all content could be covered within the allotted 2-hour classes. Over the course of the 8-week program, three meditation techniques were taught to participants, with just one technique practiced during each 20-minute home practice session (typically, MBSR prescribes $40 \mathrm{~min} / \mathrm{d}$ of practice; however, this was reduced to 20 minutes to match the home practice prescribed by the RR program). During the first 2 weeks, participants practiced mindful bodyscan; during weeks 3 and 4 , they practiced mindful yoga (simple yoga postures done with a mindful attitude); and then during weeks 5 to 6 , they practiced sitting meditation (which comprises watching the flow of breath and mental phenomena without trying to control these). During the final 2 weeks, participants were free to choose which practice to do. The MBSR course was taught by Carol Legro, who completed the MBSR teacher training program and has more than 12 years experience teaching MBSR. See Appendix C, Supplemental Digital Content, http://links.lww.com/PSYMED/A474, for further details about the courses.

\section{Experimental Design}

\section{Behavioral Measures}

The following questionnaires were administered at baseline and postprograms.

\section{The Perceived Stress Scale}

The Perceived Stress Scale (PSS) (42) assesses the degree to which situations are appraised as stressful (i.e., unpredictable, uncontrollable, and overloading) and was our main clinical outcome measure. The scale in cludes 10 items, that is, "How often have you felt confident about your ability to handle your personal problems?" which participants respond to on a five-point Likert scale from 0 being never to 4 being very often. Total scores range from 0 to 40 , with higher scores indicating greater overall distress and $\alpha$ reliability coefficient ranges from .75 to .86 .

\section{The Five Facet Mindfulness Questionnaire}

The Five Facet Mindfulness Questionnaire (FFMQ) (43) is a commonly used, 39-item measure consisting of five subscales (observing, describing, acting with awareness, nonjudging of inner experience, and nonreactivity to inner experience). Observing includes noticing or attending to internal and external experiences such as sensations, thoughts, or emotions. Describing denotes labeling internal experiences with words. Acting with awareness refers to focusing on one's activities in the moment as opposed to behaving mechanically. Nonjudgment of inner experience refers to taking a nonevaluative stance toward thoughts and feelings. Nonreactivity to inner experience is allowing thoughts and feelings to come and go, without getting caught up in them. The scale includes 39 sentences such as "I perceive my feelings and emotions without having to react to them," and the participants are asked to rate on a Likert-type scale ranging from 1 (never or very rarely true) to 5 (very often or always true). The FFMQ contains both positively and negatively worded items ( 20 and 19 , respectively), with higher scores indicating higher levels of mindfulness. $\alpha$ Reliability coefficient ranges from .67 to .93

\section{The Self-Compassion Scale}

The Self-Compassion Scale (44) includes items that measure how often people respond to feelings of inadequacy or suffering with self-kindness (e.g., "I try to be loving toward myself when I'm feeling emotional pain"), self-judgment (e.g., "I'm disapproving and judgmental about my own flaws and inadequacies"), common humanity (e.g., "I try to see my failings as part of the human condition"), isolation (e.g., "When I think about my inadequacies it tends to make me feel more separate and cut off from the rest of the world"), mindfulness (e.g., "When something painful happens I try to take a balanced view of the situation"), and overidentification (e.g., "When I'm feeling down I tend to obsess and fixate on everything that's wrong"). The scale includes 26 items, rated on a 5-point scale ranging from 1 (almost never) to 5 (almost always). $\alpha$ Reliability coefficients range from .75 to .81 .

\section{The Rumination Response Questionnaire}

The Rumination Response Questionnaire (45) assesses depressive rumination styles and measures the tendencies to a) reflect on problems, defined as contemplative, intentional pondering of one's mood with a focus on problem solving and b) brood, referring to passive and judgmental pondering of one's mood. The scale includes 22 items, such as "I think "why do I have problems other people don't have'?" and the items rated on a scale from ranging from 1 (almost never) to 4 (almost always). $\alpha$ Reliability coefficients range from .72 to .77 .

\section{Common Life Stressors}

The Holmes-Rahe Scale (46) was administered to ensure that changes in stress were not due to a recent or upcoming life event. This scale, also called the social readjustment rating scale, includes 43 life events such as "death of a close family member" or "personal injury or illness." Each event is assigned a "Life Change Unit" score, and an overall score is obtained by adding them to predict illness.

\section{Home Practice}

Participants were given paper logs on which to record their daily meditation practice. Compliance is listed in Table 1 along with attendance.

\section{MRI Acquisition Parameters}

All participants were scanned at the Martinos Center for Biomedical Imaging in Boston, MA. High-resolution MRI data were acquired using a Siemens Magnetom Avanto 1.5-T scanner with standard head coil. Data sets of the whole brain were collected using a T1-weighted MPRAGE sequence, consisting of 128 sagittal slices $(1.0 \times 1.0 \times 1.3 \mathrm{~mm}$; TI $=1000$ milliseconds; echo time $=3.39$ milliseconds; repetition time $(\mathrm{TR})=2730$ milliseconds). Functional data were acquired using an ascending sequence order $(\mathrm{TR}=$ 2000 milliseconds; flip angle $=90^{\circ}$; matrix size $=64 \times 64$; field of view $=200 \mathrm{~mm} ; 25$ axial slices; slice thickness $=3.0 \mathrm{~mm}$ ) Head motion was restricted using two padded clamps.

\section{MRI-Guided Meditation Sessions}

All participants underwent a 24-minute scan during which they listened to a guided recording containing excerpts from their daily home meditation practice instructions. The scan comprised four 6-minute segments, each containing verbal prompts. The prompts at each segment were matched for duration and on-set time to minimize confounds due to listening (see Fig. 1 for a graph depicting scanning sessions for each group and for exact timings of the verbal prompts and Appendix B in Supplemental Digital Content, http://links.lww.com/PSYMED/A474). For both groups, the first two segments comprised a guided resting state and a guided silent random number generation task. The third segment was the guided relaxing or mindful bodyscan, and the fourth segment contained other meditation instructions unique to each program (not included in the current analyses). The instructions were communicated through headphones, which also muffled the sound of the scanner.

The bodyscan meditation recordings were matched in terms of the body area toward which participant's attention was directed and timing onsets. The RR group received specific instructions to relax that body area, whereas the MBSR group received instructions to notice and be aware of the same body area. For instance, RR participants heard: "Now bringing awareness to your jaw, and as you exhale release any tension or clenching." "Now feeling a wave of relaxation spreading down over your face, letting your cheeks release and soften so your whole face feels comfortable and relaxed." The MBSR group heard: "Be aware of the jaw, the hinge joint on either side of the face, and the muscles of the jaw." "Bringing attention to the cheeks, the muscles of the cheeks and face that give expression to our many emotions, the sinuses, the ears, the outer ear, the ability to hear at this moment." In the present study, we specifically analyzed the bodyscan versus resting state contrasts within and between groups because the differences in ideology were likely to be most evident during bodyscan 


\section{Relaxation Response Program}

\begin{tabular}{|l|l|l|l|}
\hline Rest & $\begin{array}{l}\text { Relaxing } \\
\text { bodyscan } \\
\text { meditation }\end{array}$ & $\begin{array}{l}\text { Number } \\
\text { generation }\end{array}$ & $\begin{array}{l}\text { Focused } \\
\text { attention } \\
\text { meditation }\end{array}$ \\
\hline
\end{tabular}

\section{Mindfulness-Based Stress Reduction Program}

\begin{tabular}{|l|l|l|l|}
\hline & $\begin{array}{l}\text { Relaxing } \\
\text { bodyscan } \\
\text { meditation }\end{array}$ & $\begin{array}{l}\text { Number } \\
\text { generation }\end{array}$ & $\begin{array}{l}\text { Open } \\
\text { awareness } \\
\text { meditation }\end{array}$ \\
\hline
\end{tabular}

FIGURE 1. Study design.

\section{Scanning duration $=24$ minutes $(6$ minutes for each session $\times 4)$}

meditations and because they were matched for the focus of attention on the body.

\section{Data Analyses}

Preprocessing included correction for head motion, temporal and spatial normalization in Montreal Neurological Institute space, and smoothing using a Gaussian kernel with an isotropic kernel with a full width at half maximum of $6 \mathrm{~mm}$ using the standard pipeline for volume-based analyses provided in the Connectivity Toolbox (CONN) (47) and Statistical Parametric Mapping (SPM8; Welcome Department of Cognitive Neurology).

To ensure that participants were following the bodyscan instructions, we first performed an event-related partial least squares analysis $(48,49)$ time locked to the period when participants were attending to their face as a manipulation check. We chose this body area because this part of the recording was the longest in duration and enabled highest power to detect neural activation. Within this time course, we arbitrarily created five blocks of four TRs and averaged across these blocks. A similar procedure was applied to the resting state scan.

To test our neural hypotheses, seed-based functional connectivity analysis was performed using the CONN toolbox and the full-resting and meditation-state blocks. For time and frequency decomposition, a bandpass filter (0.01-0.15) was applied. To address artifacts such as spiking and motion, which might contribute to spurious correlations, artifact detection was used, as implemented in the CONN toolbox. CONN implements the CompCor method (50) to identify principal components associated with segmented white matter and cerebrospinal fluid for each participant. White matter, cerebrospinal fluid, and realignment parameters are entered as confounds in a first-level analysis, and the data are band-pass filtered to 0.008 to $0.09 \mathrm{~Hz}$. This method addresses the confounding effects of participant movement, without regressing the global signal and without affecting intrinsic functional connectivity (51). In addition to accounting for head motion and other nuisance factors at the single-subject level in all connectivity analyses, we also extracted maximum voxel displacement values for each participant and conducted a statistical analysis for between-group differences. The results demonstrated that there were no differences between groups in terms of average realignment values, $t(32)=0.83$ (two-sided $p=.41$ ).

For the investigation of shared functional coupling, an a priori seed in ventral medial frontal cortex (vMPFC; MNI coordinates: $-4,50,-20$ ) and an SPM "conjunction null" analysis were used. As an exploratory investigation, the same seed was used to investigate the relationship between differences in perceived stress levels and functional connectivity of the seed to whole brain during the bodyscan $>$ rest for both groups. For the investigation of differential functional coupling patterns, a priori seeds of right inferior frontal gyrus (rIFG) pars opercularis (MNI coordinates: 54, 14, 16) and the dorsal aINS (MNI coordinates: 32, 20, 0) were used. Further analyses were conducted to investigate differences in perceived stress level and functional connectivity of these seeds to whole brain during the bodyscan $>$ rest for each group. All imaging analyses were corrected for multiple comparisons using a combination of voxel-level thresholds $(p<.001)$ and cluster extent threshold with a family-wise error (FWE) rate at $p<.05$. Given the conservative nature of conjunction analysis (52), for the conjunction analysis, only data with a $p$ value less than .001 (uncorrected) are reported. For all seeds, the parcellation scheme of Hashmi et al. (53), based on the Harvard Oxford Atlas, was used. Statistical analyses compared functional connectivity differences between RR and MBSR bodyscan meditations using bodyscan $>$ rest contrasts. The results reflect connectivity of region of interests to whole brain and all coordinates reported refer to peak activations in anatomical MNI space.

\section{RESULTS}

\section{Demographics}

There were no significant differences between the RR and MBSR groups on baseline demographic characteristics or on recent life stressors (Table 1). There were significant differences in baseline perceived stress levels between the participants who attended RR $(14.10 \pm 6.5)$ and MBSR $(18.59 \pm 6.75)$ programs. However, there was no significant difference in baseline perceived stress levels between the RR $(14.69 \pm 7.0)$ and MBSR $(18.33 \pm 6.26)$ programs among participants included in the brain analyses $(t(32)=1.60, p=.12)$.

\section{Perceived Stress}

A mixed two-way analysis of variance was conducted on PSS scores including group (RR versus MBSR) as the between-subject 


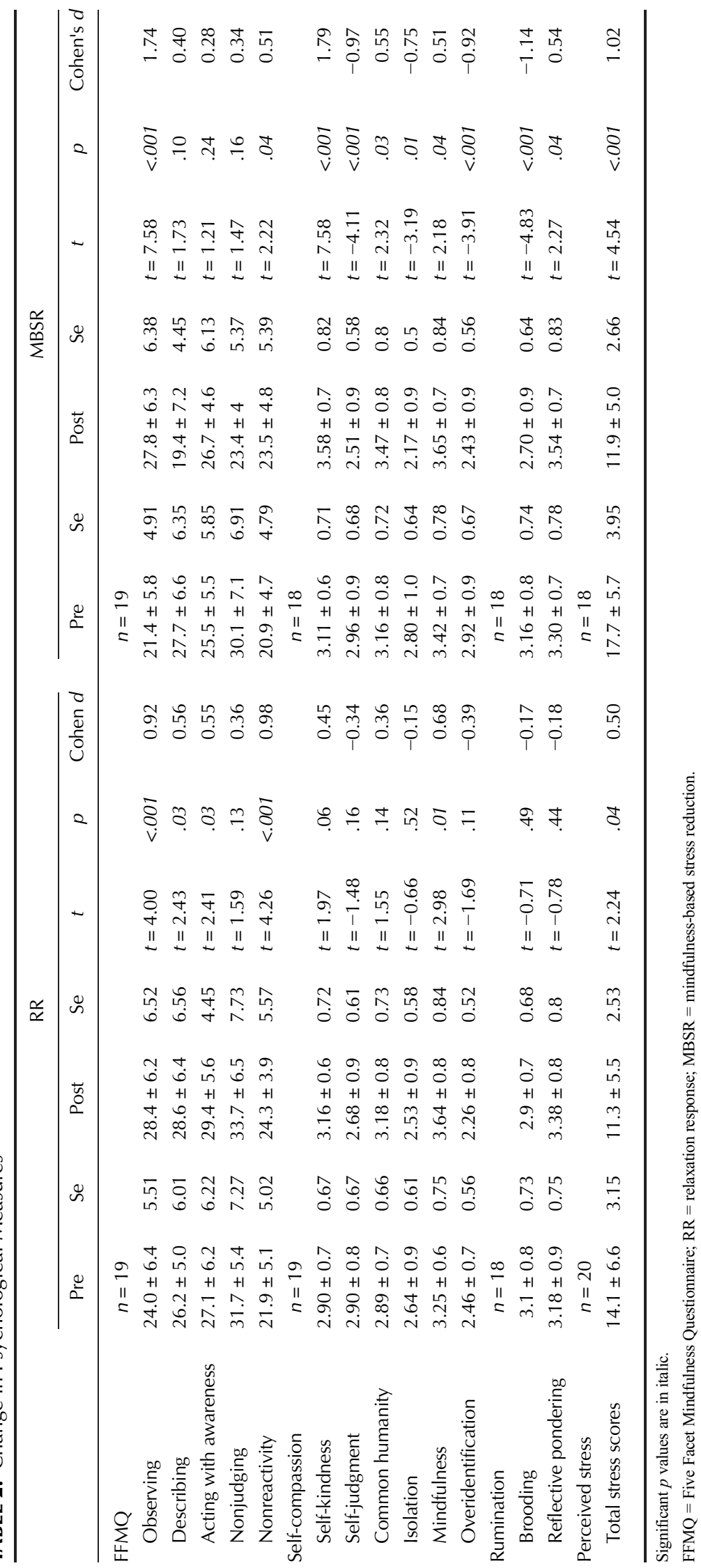


and time (pre versus post program) as the within-subject independent variable. A main effect was found for time $(F(1,37)=23.12$, $p<.001)$, indicating that both programs were successful in reducing perceived stress. As expected, the group by time interaction was not significant $(F(1,37)=2.80, p=.10)$, indicating that groups did not differ in the reduction of perceived stress. Because neuroimaging data were only available from a subset of participants, we repeated the same analyses including only those participants with neuroimaging data. Similar results were obtained, indicating a main effect for time $(F(1,30)=19.00, p<.001)$ and a nonsignificant group by time interaction $(F(1,30)=1.51, p=.21)$.

\section{Other Behavioral Measures}

The effects of each program on psychological measures are listed in Table 2. The RR program was associated with increases in the "describing," "acting with awareness," "observing," and "nonreactivity" facets of the FFMQ. The MBSR program was associated with increases in the observing and nonreactivity facets of the FFMQ, as well as increases in self-compassion and decreases in rumination levels. A series of analyses of variance comparing pre-post differences in these measures between groups did not yield significant results (maximal $F(1,37)=2.801, p=.10$ ), indicating that change in these measures did not significantly differ between groups. There was a correlation between change in PSS scores and change in the self-kindness subscale in MBSR $(r=-0.568, p<.05)$ and between change in PSS and the common humanity subscale of the Self-Compassion Scale in RR ( $r=-0.493$, $p<.05)$. There were no correlations between change in PSS scores and changes on other measures for either group.

\section{Analysis of Bodyscan Instructions}

To confirm that the two stress reduction programs differed in terms of their emphasis on relaxation and awareness, we assessed the frequency of instructions that included awareness or relaxation components in the home practice guided meditation instructions. On average, for the RR bodyscan recording, $78 \%$ of the instructions alluded to relaxation or feeling relaxed and $68.3 \%$ alluded to awareness. For the MBSR recording, 37.5\% alluded to relaxation or feeling relaxed and $84.4 \%$ alluded to awareness. The differences in instructions that included awareness and relaxation components were statistically significant - instructions with relaxation components were more frequent in RR $(p<.001$, Fisher's exact test), and the instructions with awareness components were more frequent in MBSR $(p<.01$, Fisher's exact test).

\section{Neuroimaging Results}

Before testing our hypotheses, we first performed a manipulation check to ensure that participants were complying with the guided meditation instructions. An event-related analysis of bodyscan $>$ rest was performed time locked to the period when participants were focused on their face. As detailed in Methods, this particular location was chosen because it was the body area with the longest duration, enabling averaging across several TRs. This revealed a significant cluster in primary somatosensory cortex $(+66,-12,+28 ; 12$ voxels, cluster $p<.001)$ in an area frequently associated with the face (Neurosynth, retrieved on June 29, http://neurosynth.org/locations/66_-12_28_6/), suggesting that participants were complying with the meditation instructions.

To investigate patterns of functional coupling shared across the two bodyscan meditations, "conjunction null" analyses were performed using individual RR bodyscan > rest and MBSR bodyscan $>$ rest contrasts in SPM8. Analysis of RR bodyscan $>$ rest contrast using a vMPFC seed yielded a significant cluster with the peak coordinates $(+50,-78,-02 ; 74$ voxels, cluster $p$-FWE $=$ $.018)$ in extrastriate area, whereas analysis of MBSR bodyscan $>$ rest contrast using vMPFC seed yielded two significant clusters with peak coordinates $(+2,+30,+48 ; 82$ voxels, cluster $p$-FWE $<.001)$ and $(+16,-88,-10 ; 61$ voxels, cluster $p$-FWE $=.045)$ in prefrontal and occipital cortices, respectively. Conjunction analysis using these contrasts revealed a significant cluster within supplementary motor areas with the peak coordinates $(+2,+34,+38 ; 43$ voxels, $p<.001$, false discovery rate cluster corrected at $p<.05$; Fig. 2). Additional analyses examined the relationship between functional connectivity during bodyscan (bodyscan meditation $>$ rest contrast) and differences in perceived levels of stress from printervention to postintervention with all participants. Whole-brain correlation between vMPFC connectivity and improvement in stress revealed a significant cluster in frontopolar cortex with peak coordinates $(+34,+58,+02 ; 74$ voxels, cluster $p$-FWE $<.05)$.

To test our hypothesis regarding the differential engagement of rIFG in RR versus MBSR, whole-brain functional connectivity disjunction analyses were performed using the rIFG seed for the bodyscan versus rest contrast. The RR group demonstrated differential coupling of rIFG regions with anterior cingulate cortex (ACC), supplementary motor areas (SMA), and aINS (Fig. 3A, Table 3A). No significant regions were identified in the MBSR group. A between-group analysis of the [bodyscan $>$ rest] contrasts yielded a significant cluster in supplementary and presupplementary motor areas for the RR group in both hemispheres with the peak coordinates $(-04,+04,+50 ; 194$ voxels, cluster $p$-FWE $<.001$; Fig. 3B).

Next, whole-brain functional connectivity analyses were performed using the aINS seed. The disjunction analyses for the MBSR versus RR comparison using [bodyscan > rest] contrasts did not reach significance (all $p>.05$, FWE corrected). The analysis for the RR group alone did not yield significant results. The analysis for only the MBSR group demonstrated coupling of aINS with pregenual anterior cingulate cortex (pACC) and precentral gyrus (Fig. 4, Table 3B). The analysis investigating differences in perceived stress levels and functional connectivity of these seeds to whole brain during the bodyscan $>$ rest for each group did not yield significant results.

\section{DISCUSSION}

As expected, both the RR and MBSR programs were associated with comparable decreases in perceived stress levels but differed in psychological measures of mindfulness, rumination, and selfcompassion. In addition, the two programs exhibited common and dissociable functional coupling patterns during their respective bodyscan meditations. Functional coupling of vMPFC to supplementary motor area was shared across the two types of bodyscan meditations, potentially reflecting the present moment awareness and focused attention to the body that is integral to both bodyscan meditations. The functional coupling of this seed to a cluster in 


\section{A}

\section{Relaxing and mindful body-scan meditation > rest}

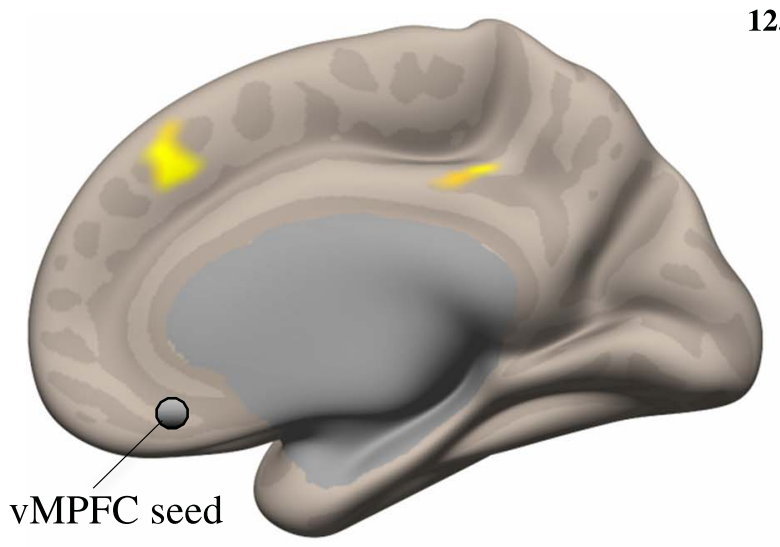

12.00

$\mathbf{0 . 0 0}$

B

Neural correlates of differences in perceived stress during the relaxing and mindful body-scan meditations $>$ rest
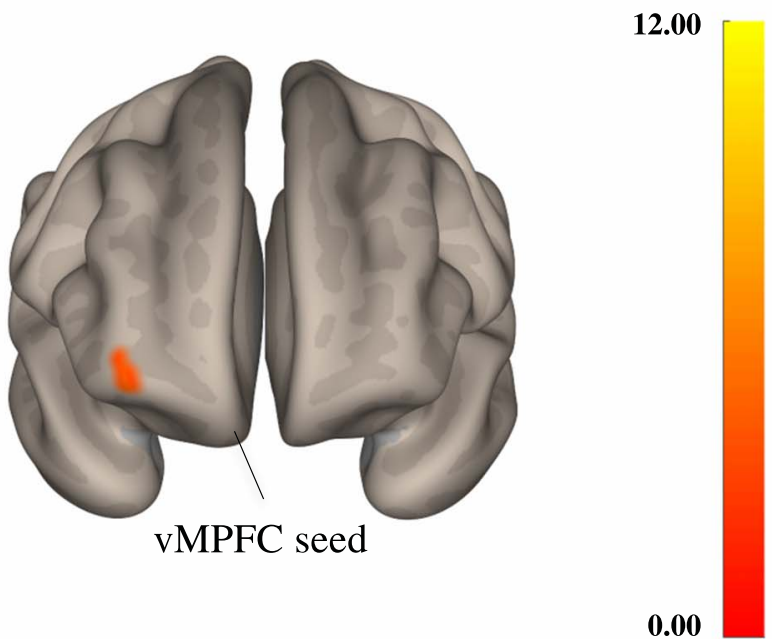

FIGURE 2. A, Functional coupling of the vMPFC seed (circled in gray; bodyscan > rest contrast). Conjunction analysis revealed a significant cluster within supplementary motor areas with the peak coordinates $(+2,+34,+38 ; 43$ voxels, false discovery rate cluster corrected at $p<.05)$. B, Functional coupling of the vMPFC seed (bodyscan $>$ rest contrast) in relation to the differences in perceived stress across all participants. $\mathrm{vMPFC}=$ ventral medial frontal cortex; FDRc $=\mathrm{X}$.

TABLE 3. Functional Coupling Patterns for the RR and MBSR Bodyscan Meditations

\begin{tabular}{|c|c|c|c|c|c|}
\hline Region & Hem & Clusters $(x, y, z)$ & Size & $p$-FWE & $p-\mathrm{FDR}$ \\
\hline \multicolumn{6}{|c|}{ A. Peak coordinates of clusters functional coupled to rIFGpo during a relaxing bodyscan $>$ rest for the RR group } \\
\hline SMA & $\mathrm{L}$ & $-06,-04,+56$ & 191 & .001 & .001 \\
\hline ACC & $\mathrm{L}$ & $-08,+36,+28$ & 82 & .002 & .001 \\
\hline INS & $\mathrm{R}$ & $+34,+18,+02$ & 45 & .06 & .03 \\
\hline \multicolumn{6}{|c|}{ B. Peak coordinates of clusters functional coupled to aINS during a mindful bodyscan > rest for the MBSR group } \\
\hline sACC & $\mathrm{R}$ & $+04,+28,-08$ & 64 & .03 & .03 \\
\hline Pre_C & $\mathrm{R}$ & $+66,+04,+20$ & 56 & .06 & .03 \\
\hline
\end{tabular}

$\mathrm{RR}=$ relaxation response; $\mathrm{MBSR}=$ mindfulness-based stress reduction; $\mathrm{SMA}=$ supplementary motor area; $\mathrm{ACC}=$ anterior cingulate cortex; INS $=$ insula; FWE = family-wise error rate; FDR = false discovery rate; rIFGpo = inferior frontal gyrus pars opercularis; aINS = anterior insula; $\mathrm{sACC}=$ subgenual ACC; Pre_C $=$ precentral gyrus. 
A

\section{Relaxing body-scan meditation $>$ rest}
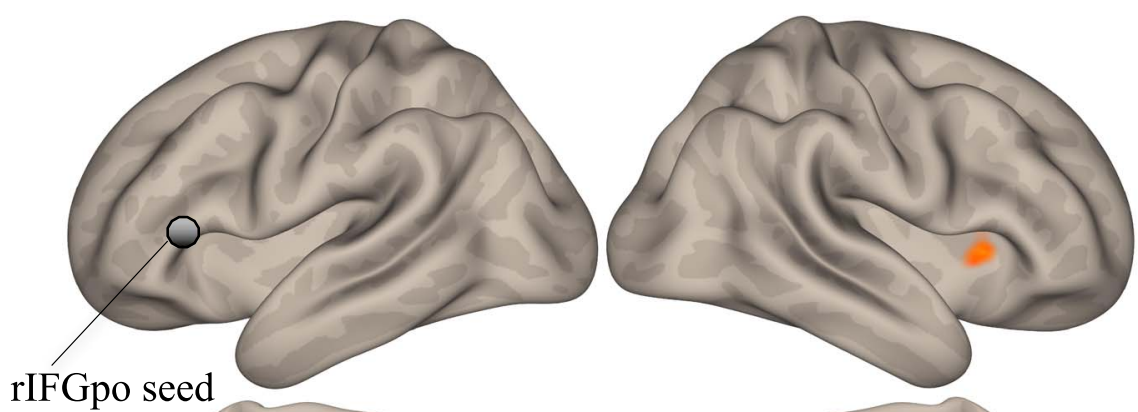

12.00
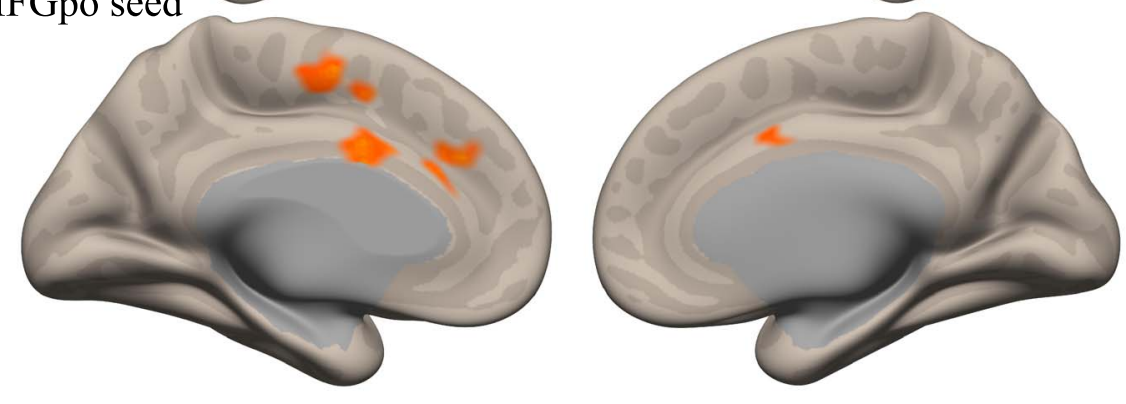

B

\section{Relaxing body-scan meditation $>$ rest vs. mindful body-scan meditation $>$ rest}
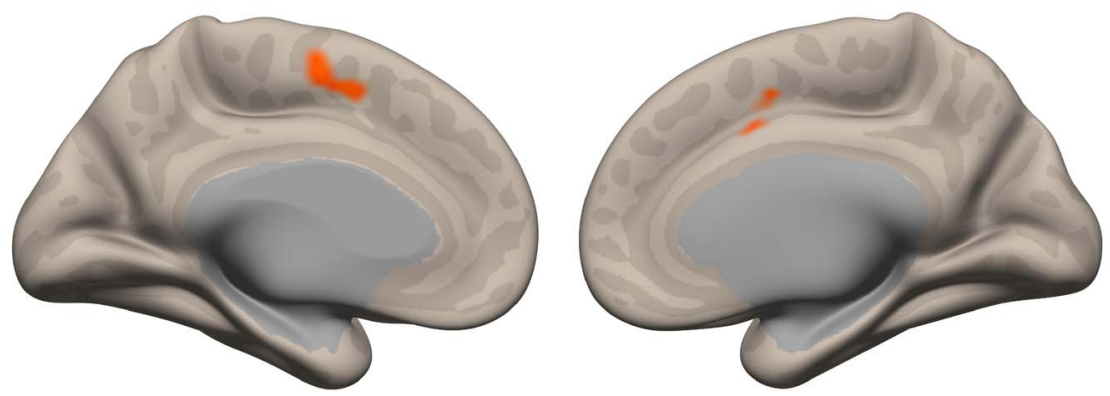

FIGURE 3. A, Functional coupling of rIFGpo (circled in gray) for the RR group (bodyscan > rest contrast). The results demonstrated coupling of rIFG regions with ACC, SMAs, and aINS during RR bodyscan. B, Differences between the functional coupling of rIFG seed to whole brain between the RR and the MBSR groups (bodyscan > rest; Table 3A). A between-group analysis of the [bodyscan $>$ rest] contrasts yielded a significant cluster in supplementary and presupplementary motor areas for the RR group in both hemispheres with the peak coordinates $(-04,+04,+0 ; 194$ voxels, cluster $p$-FWE $=.000067)$. rIFGpo $=$ inferior frontal gyrus pars opercularis; $\mathrm{RR}=$ relaxation response; $\mathrm{rIFG}=$ right inferior frontal gyrus; $\mathrm{MBSR}=$ mindfulness-based stress reduction.

frontopolar cortex correlated with reductions in perceived stress levels. The RR bodyscan was differentially associated with specific neural activity in regions associated with control of physical/autonomic relaxation (rIFG and SMA). Moreover, although regions commonly associated with interoceptive awareness (aINS and pACC) were functionally coupled during the MBSR bodyscan, this pattern did not differentiate the mindful from the relaxing bodyscan. These data highlight that specific theoretical orientations that are incorporated into guided meditation practices can influence neural activity, which in turn could potentially mediate differential clinical outcomes.

Both stress reduction courses were associated with decreases in perceived stress and increases in levels of mindfulness as measured by the FFMQ. Interestingly, although both programs were associated with significant increases in the "observing" and "nonreactivity" subscales of the FFMQ, the RR program further resulted in significant increases in the "describing" and "acting with awareness" subscales. The RR program introduces the concept of mindfulness and includes 2 minutes of mindful breathing during the daily-guided home meditation practice. Although we anticipated that there might be some increase in mindfulness in the RR group, we had hypothesized that the mindfulness changes would be larger in the MBSR group, contrary to our findings. Although it is possible that changes in mindfulness are indeed larger in the RR program, the FFMQ is 


\section{Mindful body-scan meditation $>$ rest}

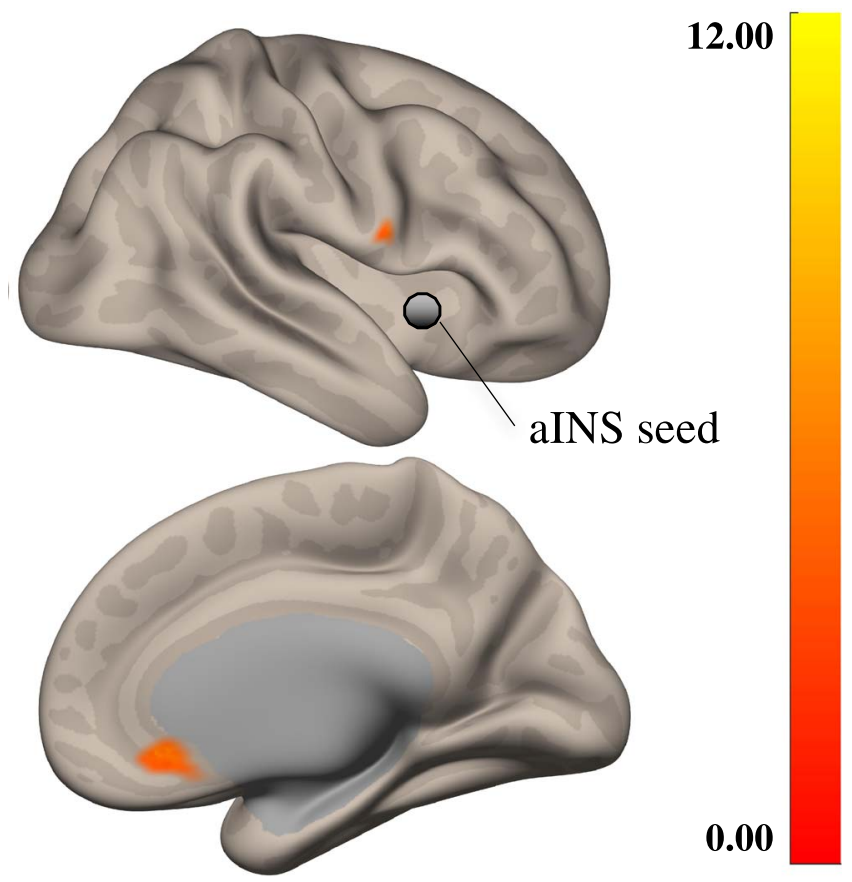

FIGURE 4. Functional coupling of aINS (circled in green) during a mindful bodyscan compared with rest for the MBSR group. The results revealed functional coupling of aINS with pACC and precentral gyrus (Table 3B). aINS = anterior insula; MBSR = mindfulness-based stress reduction; $\mathrm{pACC}=$ pregenual anterior cingulate cortex.

known to have several psychometric issues with respect to it's factor structure (54), which might also explain this discrepancy. Further research using other methods to assess mindfulness will be necessary to reach a conclusion with respect to the relative role of mindfulness in the two programs.

Only MBSR was associated with changes in self-compassion and rumination. Previous studies have found mediating relationships between mindfulness, self-compassion, and rumination and clinical outcome measures in the MBSR programs $(13,40,55,56)$. To our knowledge, changes in compassion and rumination have not been previously assessed in relation to the RR program. The differences in these psychological metrics suggest that each program may reduce perceived stress through differential psychological processes. Although correlations were not found between most of these metrics and levels of stress reduction and/or brain connectivity, the lack of significant results could be due to our small sample size, which limits the determination of dissociable functional coupling patterns associated with each stress reduction program. It is also important to note that each program uses multiple meditation techniques and both programs include didactic content that was not matched between the programs. These other program elements likely provide additional components, although the founders of both programs have explicitly stated that all aspects of their program are designed to foster increased relaxation or mindfulness, respectively. For the present study, we wished to study the existing RR and MBSR programs because each is well validated, widely used, and well studied. Future larger studies examining programs that have identical didactic content and specifically focus on one meditation technique per program are needed to more precisely investigate the differential effects.
Both styles of bodyscan meditation shared functional coupling patterns between vMPFC and supplementary motor areas, which is consistent with focused attention on bodily sensations and present-centered awareness of the self (26). Mindfulness training has been previously associated with vMPFC, especially in relation to modulation of value signals (57). Functional coupling between these two regions has also previously been associated with cognitive control (58). Moreover, the correlation between functional connectivity of the vMPFC and differences in perceived levels of stress yielded a significant cluster in frontopolar cortex. Gray matter in this region was found to be thicker in advanced mindfulness meditation practitioners compared with nonmeditators (33). The functional coupling between these regions has also been previously associated with individual differences in behavioral flexibility (59). Flexibility is critical for coping and adjustment to stressful conditions, and has been shown to improve after mindfulness meditation (60-62). Frontopolar cortex has also been implicated in cognitive control of emotion through strategies such as reappraisal and suppression $(63,64)$, as well as in goal formation and maintenance (65). The observed correlation between reductions in perceived stress and the functional coupling between these regions is consistent with these findings. However, more research is required to draw conclusions on the relationship between stress reduction and the present functional coupling patterns.

The RR program specifically emphasizes decreasing sympathetic activation through a deliberate reduction of arousal and muscle tension (66). After training, participants exhibited differential functional coupling of rIFG - an important hub of intentional inhibition and control (30,31) - to the ACC and SMA during a relaxing bodyscan compared with rest. Critically, functional coupling of 
rIFG pars opercularis to SMA differentiated the relaxing bodyscan from the mindful bodyscan, implicating inhibitory neural networks in the relaxing bodyscan meditation. The rIFG and SMA are among the areas identified in previous studies of biofeedback-mediated physical relaxation $(67,68)$, supporting the hypothesis that RR-based stress reduction may be mediated by physical relaxation during the bodyscan.

Preparation and execution of voluntary muscle relaxation has previously been associated with motor inhibition and with neural activity in primary and supplementary motor areas (69). The rIFG functions as a domain general "brake" mechanism over response tendencies $(30,31)$. This brake can be turned on both externally by salient stop signals and internally by goals. Both the rIFG and SMA have previously been implicated in motor response inhibition using stop-signal paradigms, and an rIFG-based network is thought to play a causal role in inhibitory motor control $(70,71)$. However, because these experimental paradigms usually involve multiple cognitive processes of attentional capture and response inhibition, no consensus has been achieved regarding the exact contribution of inferior frontal gyrus to attention and inhibition (72-74). Given that the two bodyscan protocols were largely identical with regard to attentional capture and differed only in terms of additional "relaxation" component, the primary contribution of the rIFG may be motor inhibition rather than attention. In line with this interpretation, prior research associated this region with motor intentions and higher-level representations that exist before and independently of action execution $(75,76)$.

During the MBSR bodyscan, which emphasizes nonjudgmental awareness to sensory experience, the right aINS - an important hub of sensory awareness and salience - was strongly coupled with pregenual ACC. The aINS is associated with awareness of the sensory aspects of experience and subjective feeling states (77) and has been identified in multiple neuroimaging studies of mindfulness meditation $(26,32,37,78)$. The aINS is part of the circuitry implicated in anxiety (79) and has projections to cingulate regions (80). The ACC is involved in emotional regulation, and the pregenual subdivision specifically regulates limbic activity during the generation of emotional responses (81). Our finding of increased functional coupling of aINS and pACC during the MBSR bodyscan may thus indicate enhanced awareness of the sensory aspects of affective experience and modulation of emotional responses to this affective experience through the neural functions of the ACC. However, it is important to note that this functional coupling pattern did not differentiate the MBSR from the RR bodyscan, possibly suggesting the presence of these processes during the $R R$ bodyscan to a certain degree.

As mentioned previously, the RR program introduces the concept of mindfulness to participants and instructs individuals to become more aware of present moment sensory experience, which is one component of mindfulness. However, during the RR bodyscan, every instruction to become aware of a body region is followed by an instruction to relax that area, whereas the MBSR bodyscan instructions are to just be aware, without trying to change anything. Interestingly, although the aINS-pACC functional coupling pattern was significant in the MBSR group, the disjunction analyses using aINS seed did not yield significant results. This is consistent with the idea that the RR program develops the skill of mindfulness.

Numerous studies have demonstrated the beneficial effects of a variety of meditation practices. It has been unclear to what extent these practices share neural correlates. Interestingly, a recent study compared electroencephalogram activity during a focused-attention and open monitoring meditation practice from practitioners of two Buddhist traditions (17). The researchers found that the differences between the two meditation traditions were more pronounced than the differences between the two types of meditation. These data are consistent with our findings that theoretical orientation of how a practice is taught strongly influences neural activity during these practices. However, the study used long-term practitioners from different cultures, which may have confounded the results. By directly comparing these programs in a homogeneous meditation-naive cohort and using the same data collection and analysis methods, we were able to confirm that the RR and MBSR programs are associated with differential psychological and neural processes. These results are in line with theoretical models that attempt to describe potential mechanisms of change associated with meditation (82-84) and suggest that different meditation practices may be associated with overlapping as well as differential neural correlates and behavioral outcomes, which may potentially have significant effects on disease. One limitation of the present study is the lack of a passive control group, which limits our ability to interpret findings. It is possible that all observed changes were due to passage of time or generic group effects. Nevertheless, prior studies with control groups have demonstrated that both programs are successful in reducing stress levels (40) and that MBSR program is effective for increasing mindfulness and compassion while decreasing rumination.

Our results suggest that these programs likely promote wellness through shared and different processes, and each intervention may therefore potentially have differential effects on treatment of illnesses. Future studies with larger sample sizes would be beneficial to assess the relationship between pre-post neural changes associated with each intervention and the behavioral outcome measures. Another limitation of the study was the cluster randomization method, which can be sensitive to intragroup correlations, seasonal effects, and intermittent environmental stressors. Finally, future studies specifically designed to compare differential effects of these programs on particular diseases will be necessary to determine if these differences need to be taken into consideration when prescribing meditation-based programs.

We thank Janet Fronk, Carol Legro, Christina Congleton, Lucas Morgan, Nicole Chung, Yuliya Kulyomina, Ilana Rosman, and Sita Yerramsetti for their help, and our study participants for their participation.

Source of Funding and Conflicts of Interest: This project was funded by R01 AG048351, R21 AT003425, S10 RR019307, S10 RR023401. There are no conflicts of interest.

\section{REFERENCES}

1. Barnes PM, Powell-Griner E, McFann K, Nahin RL. Complementary and alternative medicine use among adults: United States, 2002. Semin Integr Med 2004; 2:54-71.

2. Benson H, Greenwood MM, Klemchuk H. The relaxation response: psychophysiologic aspects and clinical applications. Int J Psychiatry Med 1975;6:87-98.

3. Hoffman JW, Benson H, Arns PA, Stainbrook GL, Landsberg GL, Young JB, Gill A. Reduced sympathetic nervous system responsivity associated with the relaxation response. Science 1982;215:190-2.

4. Beary JF, Benson H. A simple psychophysiologic technique which elicits the hypometabolic changes of the relaxation response. Psychosom Med 1974;36: $115-20$. 
5. Kabat-Zinn J. An outpatient program in behavioral medicine for chronic pain patients based on the practice of mindfulness meditation: theoretical considerations and preliminary results. Gen Hosp Psychiatry 1982;4:33-47.

6. Kabat-Zinn J. Full Catastrophe Living New York: Delta Publishing; 1990.

7. Kabat-Zinn J, Lipworth L, Burney R. The clinical use of mindfulness meditation for the self-regulation of chronic pain. J Behav Med 1985;8:163-90.

8. Samuelson M, Foret M, Baim M, Lerner J, Fricchione G, Benson H, Dusek J, Yeung A. Exploring the effectiveness of a comprehensive mind-body intervention for medical symptom relief. J Altern Complement Med 2010;16: 187-92.

9. Chiesa A, Serretti A. A systematic review of neurobiological and clinical features of mindfulness meditations. Psychol Med 2010;40:1239-52.

10. Grossman P, Niemann L, Schmidt S, Walach H. Mindfulness-based stress reduction and health benefits. J Psychosom Res 2004;57:35-43.

11. Dusek JA, Hibberd PL, Buczynski B, Chang BH, Dusek KC, Johnston JM, Wohlhueter AL, Benson H, Zusman RM. Stress management versus lifestyle modification on systolic hypertension and medication elimination: a randomized trial. J Altern Complement Med 2008;14:129-38.

12. Olson KL, Emery CF. Mindfulness and weight loss: a systematic review. Psychosom Med 2015;77:59-67.

13. Speca M, Carlson LE, Goodey E, Angen M. A randomized, wait-list controlled clinical trial: the effect of a mindfulness meditation-based stress reduction program on mood and symptoms of stress in cancer outpatients. Psychosom Med 2000;62:613-22.

14. Benson H, Beary JF, Carol MP. The relaxation response. Psychiatry 1974;37: 37-46.

15. Bhasin MK, Dusek JA, Chang BH, Joseph MG, Denninger JW, Fricchione GL, Benson H, Libermann TA. Correction: relaxation response induces temporal transcriptome changes in energy metabolism, insulin secretion and inflammatory pathways. PLoS One 2017;12:e172873.

16. Deckro GR, Ballinger KM, Hoyt M, Wilcher M, Dusek J, Myers P, Greenberg B, Rosenthal DS, Benson $\mathrm{H}$. The evaluation of a mind/body intervention to reduce psychological distress and perceived stress in college students. J Am Coll Health 2002; $50: 281-7$

17. Amihai I, Kozhevnikov M. Arousal vs. relaxation: a comparison of the neurophysiological and cognitive correlates of Vajrayana and Theravada meditative practices. PLoS One 2014;9:e102990.

18. Fox KC, Dixon ML, Nijeboer S, Girn M, Floman JL, Lifshitz M, Ellamil M, Sedlmeier P, Christoff K. Functional neuroanatomy of meditation: a review and meta-analysis of 78 functional neuroimaging investigations. Neurosci Biobehav Rev 2016;65:208-28.

19. Cahn BR, Polich J. Meditation states and traits: EEG, ERP, and neuroimaging studies. Psychol Bull 2006;132:180.

20. Carmody J, Baer RA. Relationships between mindfulness practice and levels of mindfulness, medical and psychological symptoms and well-being in a mindfulness-based stress reduction program. J Behav Med 2008;31:23-33.

21. Feldman G, Greeson J, Senville J. Differential effects of mindful breathing, progressive muscle relaxation, and loving-kindness meditation on decentering and negative reactions to repetitive thoughts. Behav Res Ther 2010;48:1002-11.

22. Birnie K, Speca M, Carlson LE. Exploring self-compassion and empathy in the context of mindfulness-based stress reduction (MBSR). Stress Health 2010;26 359-71.

23. Shapiro SL, Astin JA, Bishop SR, Cordova M. Mindfulness-based stress reduction for health care professionals: results from a randomized trial. Int J Stress Manag 2005;12:164-76.

24. Davidson RJ, Kaszniak AW. Conceptual and methodological issues in research on mindfulness and meditation. Am Psychol 2015;70:581-92

25. Tang YY, Hölzel BK, Posner MI. The neuroscience of mindfulness meditation. Nat Rev Neurosci 2015;16:213-25.

26. Farb NA, Segal ZV, Mayberg H, Bean J, McKeon D, Fatima Z, Anderson AK Attending to the present: mindfulness meditation reveals distinct neural modes of self-reference. Soc Cogn Affect Neurosci 2007;2:313-22.

27. Mooneyham BW, Mrazek MD, Mrazek AJ, Mrazek KL, Phillips DT, Schooler JW. States of mind: characterizing the neural bases of focus and mindwandering through dynamic functional connectivity. J Cogn Neurosci 2017 29:495-506.

28. Tang YY, Ma Y, Fan Y, Feng H, Wang J, Feng S, Lu Q, Hu B, Lin Y, Li J, Zhang Y, Wang Y, Zhou L, Fan M. Central and autonomic nervous system interaction is altered by short-term meditation. Proc Natl Acad Sci 2009;106:8865-70.

29. Cai W, Ryali S, Chen T, Li C-SR, Menon V. Dissociable roles of right inferio frontal cortex and anterior insula in inhibitory control: evidence from intrinsic and task-related functional parcellation, connectivity, and response profile analyses across multiple datasets. J Neurosci 2014;34:14652-67.

30. Aron AR, Robbins TW, Poldrack RA. Inhibition and the right inferior frontal cortex. Trends Cogn Sci 2004;8:170-7.

31. Aron AR, Robbins TW, Poldrack RA. Inhibition and the right inferior frontal cortex: one decade on. Trends Cogn Sci 2014;18:177-85.

32. Farb NA, Segal ZV, Anderson AK. Mindfulness meditation training alters cortical representations of interoceptive attention. Soc Cogn Affect Neurosci 2013;8:15-26.

33. Lazar SW, Kerr CE, Wasserman RH, Gray JR, Greve DN, Treadway MT McGarvey M, Quinn BT, Dusek JA, Benson H, Rauch SL, Moore CI, Fisch
B. Meditation experience is associated with increased cortical thickness. Neuroreport 2005; 16:1893-7.

34. Fox KC, Nijeboer S, Dixon ML, Floman JL, Ellamil M, Rumak SP, Sedlmeier P, Christoff K. Is meditation associated with altered brain structure? A systematic review and meta-analysis of morphometric neuroimaging in meditation practitioners. Neurosci Biobehav Rev 2014;43:48-73.

35. Lazar SW, Bush G, Gollub RL, Fricchione GL, Khalsa G, Benson H. Functional brain mapping of the relaxation response and meditation. Neuroreport 2000;11: 1581-5.

36. Ives-Deliperi VL, Solms M, Meintjes EM. The neural substrates of mindfulness: an fMRI investigation. Soc Neurosci 2011;6:231-42.

37. Lutz A, McFarlin DR, Perlman DM, Salomons TV, Davidson RJ. Altered anterior insula activation during anticipation and experience of painful stimuli in expert meditators. Neuroimage 2013;64:538-46.

38. Craig AD. Interoception: the sense of the physiological condition of the body. Curr Opin Neurobiol 2003;13:500-5.

39. Menon V, Uddin LQ. Saliency, switching, attention and control: a network model of insula function. Brain Struct Funct 2010;214:655-67.

40. Jain S, Shapiro SL, Swanick S, Roesch SC, Mills PJ, Bell I, Schwartz GE. A randomized controlled trial of mindfulness meditation versus relaxation training: effects on distress, positive states of mind, rumination, and distraction. Ann Behav Med 2007;33:11-21

41. Andrews-Hanna JR, Snyder AZ, Vincent JL, Lustig C, Head D, Raichle ME, Buckner RL. Disruption of large-scale brain systems in advanced aging. Neuron 2007:56:924-35.

42. Cohen S, Kamarck T, Mermelstein R. A global measure of perceived stress. J Health Soc Behav 1983;24:385-96.

43. Baer RA, Smith GT, Lykins E, Button D, Krietemeyer J, Sauer S, Walsh E, Duggan D, Williams JM. Construct validity of the Five Facet Mindfulness Questionnaire in meditating and nonmeditating samples. Assessment 2008;15:329-42.

44. Neff KD. The development and validation of a scale to measure self-compassion. Self Identity $2003 ; 2: 223-50$

45. Treynor W. Rumination reconsidered: a psychometric analysis. Cog Ther Res 2003;27:247-59.

46. Crandall CS. Psychophysical scaling of stressful life events. Psychol Sci 1992;3: 256-8.

47. Whitfield-Gabrieli S, Nieto-Castanon A. Conn: a functional connectivity toolbox for correlated and anticorrelated brain networks. Brain Connect 2012;2:125-41

48. McIntosh AR, Lobaugh NJ. Partial least squares analysis of neuroimaging data: applications and advances. Neuroimage 2004;23(Suppl 1):S250-63.

49. Krishnan A, Williams LJ, McIntosh AR, Abdi H. Partial Least Squares (PLS) methods for neuroimaging: a tutorial and review. Neuroimage 2011;56:455-75.

50. Behzadi Y, Restom K, Liau J, Liu TT. A component based noise correction method (CompCor) for BOLD and perfusion based fMRI. Neuroimage 2007; 37:90-101

51. Chai XJ, Castañón AN, Ongür D, Whitfield-Gabrieli S. Anticorrelations in resting state networks without global signal regression. Neuroimage 2012; 59:1420-8

52. Friston KJ, Penny WD, Glaser DE. Conjunction revisited. Neuroimage 2005;25: $661-7$.

53. Hashmi JA, Kong J, Spaeth R, Khan S, Kaptchuk TJ, Gollub RL. Functional network architecture predicts psychologically mediated analgesia related to treatment in chronic knee pain patients. J Neurosci 2014;34:3924-36.

54. Van Dam NT, Hobkirk AL, Danoff-Burg S, Earleywine M. Mind your words: positive and negative items create method effects on the Five Facet Mindfulness Questionnaire. Assessment 2012;19:198-204.

55. Svendsen JL, Kvernenes KV, Wiker AS, Dundas I. Mechanisms of mindfulness: rumination and self-compassion. Nordic Psychol 2017;69:71-82.

56. Carlson LE, Speca M, Patel KD, Goodey E. Mindfulness-based stress reduction in relation to quality of life, mood, symptoms of stress, and immune parameters in breast and prostate cancer outpatients. Psychosom Med 2003;65:571.

57. Kirk U, Gu X, Harvey AH, Fonagy P, Montague PR. Mindfulness training modulates value signals in ventromedial prefrontal cortex through input from insular cortex. Neuroimage 2014;100:254-62.

58. Yu J, Tseng P, Hung DL, Wu SW, Juan CH. Brain stimulation improves cognitive control by modulating medial-frontal activity and preSMA-vmPFC functional connectivity. Hum Brain Mapp 2015;36:4004-15.

59. Boorman ED, Behrens TE, Woolrich MW, Rushworth MF. How green is the grass on the other side? Frontopolar cortex and the evidence in favor of alternative courses of action. Neuron 2009;62:733-43.

60. Moore A, Malinowski P. Meditation, mindfulness and cognitive flexibility. Conscious Cogn 2009; 18:176-86.

61. Greenberg J, Reiner K, Meiran N. "Mind the trap": mindfulness practice reduces cognitive rigidity. PLoS One 2012;7:e36206.

62. Heeren A, Van Broeck N, Philippot P. The effects of mindfulness on executive processes and autobiographical memory specificity. Behav Res Ther 2009;47: 403-9.

63. Ochsner KN, Silvers JA, Buhle JT. Functional imaging studies of emotion regulation: a synthetic review and evolving model of the cognitive control of emotion. Ann N Y Acad Sci 2012;1251:E1-24. 
64. Hare TA, Camerer CF, Rangel A. Self-control in decision-making involves modulation of the vmPFC valuation system. Science 2009; 324:646-8.

65. Koechlin E, Hyafil A. Anterior prefrontal function and the limits of human decision-making. Science 2007;318:594-8.

66. Wallace RK, Benson H, Wilson AF. A wakeful hypometabolic physiologic state. Am J Physiol 1971;221:795-9.

67. Critchley HG, Wiens S, Rotshtein P, Ohman A, Dolan RJ. Neural systems supporting interoceptive awareness. Nat Neurosci 2004;7:189-95.

68. Critchley HD, Corfield DR, Chandler MP, Mathias CJ, Dolan RJ. Cerebral correlates of autonomic cardiovascular arousal: a functional neuroimaging investigation in humans. J Physiol 2000;523:259-70.

69. Toma K, Honda M, Hanakawa T, Okada T, Fukuyama H, Ikeda A, Nishizawa S, Konishi J, Shibasaki H. Activities of the primary and supplementary motor areas increase in preparation and execution of voluntary muscle relaxation: an eventrelated fMRI study. J Neurosci 1999;19:3527-34

70. Wessel JR, Aron AR. Inhibitory motor control based on complex stopping goals relies on the same brain network as simple stopping. Neuroimage 2014; 103:225-34

71. Wessel JR, Conner CR, Aron AR, Tandon N. Chronometric electrical stimulation of right inferior frontal cortex increases motor braking. J Neurosci 2013;33 19611-9.

72. Hampshire A, Chamberlain SR, Monti MM, Duncan J, Owen AM. The role of the right inferior frontal gyrus: inhibition and attentional control. Neuroimage 2010;50:1313-9.

73. Duann JR, Ide JS, Luo X, Li CR. Functional connectivity delineates distinct roles of the inferior frontal cortex and presupplementary motor area in stop signal inhibition. J Neurosci 2009;29:10171-9.
74. Sharp DJ, Bonnelle V, De Boissezon X, Beckmann CF, James SG, Patel MC, Mehta MA. Distinct frontal systems for response inhibition, attentional capture, and error processing. Proc Natl Acad Sci U S A 2010;107:6106-11.

75. Roskies AL. How does neuroscience affect our conception of volition? Annu Rev Neurosci 2010;33:109-30.

76. Haggard P. Human volition: towards a neuroscience of will. Nat Rev Neurosci 2008;9:934-46.

77. Craig AD. How do you feel-now? The anterior insula and human awareness. Nat Rev Neurosci 2009;10:59-70.

78. Gard T, Hölzel BK, Sack AT, Hempel H, Lazar SW, Vaitl D, Ott U. Pain attenuation through mindfulness is associated with decreased cognitive control and increased sensory processing in the brain. Cereb Cortex 2012;22:2692-702.

79. Grupe DW, Nitschke JB. Uncertainty and anticipation in anxiety: an integrated neurobiological and psychological perspective. Nat Rev Neurosci 2013;14:488-501.

80. Augustine JR. Circuitry and functional aspects of the insular lobe in primates including humans. Brain Res Brain Res Rev 1996;22:229-44.

81. Etkin A, Egner T, Kalisch R. Emotional processing in anterior cingulate and medial prefrontal cortex. Trends Cogn Sci 2011;15:85-93.

82. Taren AA, Gianaros PJ, Greco CM, Lindsay EK, Fairgrieve A, Brown KW, Rosen RK, Ferris JL, Julson E, Marsland AL, Creswell JD. Mindfulness meditation training and executive control network resting state functional connectivity: a randomized controlled trial. Psychosom Med 2017;79:674-83.

83. Hölzel BK, Lazar SW, Gard T, Schuman-Olivier Z, Vago DR, Ott U. How does mindfulness meditation work? Proposing mechanisms of action from a conceptual and neural perspective. Perspect Psychol Sci 2011;6:537-59.

84. Vago DR, Silbersweig DA. Self-awareness, self-regulation, and self-transcendence (S-ART): a framework for understanding the neurobiological mechanisms of mindfulness. Front Hum Neurosci 2012;6:296. 